\title{
Optimal selection of pumps as turbines for maximizing electrical energy production
}

\author{
Lucrezia Manservigi ${ }^{1 *}$, Mauro Venturini ${ }^{1}$ and Enzo Losi ${ }^{1}$ \\ ${ }^{1}$ Dipartimento di Ingegneria, Università degli Studi di Ferrara, 44122 Ferrara, Italy
}

\begin{abstract}
A Pump as Turbine (PAT) is a renewable energy technology that can be a cost-effective and reliable alternative to hydraulic turbines in micro and small hydropower plants. In order to further favour PAT exploitation, a general procedure that allows the identification of the most suitable turbomachine to install is required. To this purpose, this paper develops a novel methodology aimed at selecting the best PAT that, among several alternatives, maximizes energy production. The methodology comprises two steps, which only require the knowledge of the best efficiency point of the considered pump and the hydraulic parameters of the site. The novel methodology is validated in this paper by calculating the electrical energy production of a simulated water distribution network coupled with several PATs, whose performance curves, both in direct and reverse modes, are taken from the literature. For the sake of generality, the considered turbomachines account for different geometrical characteristics, rotational speeds and operating ranges.
\end{abstract}

\section{Introduction}

Micro and small hydropower plants are one of the most important renewable energy resources in small localities, OECD countries, rural and remote areas. However, they require alternative hydro generating systems compared to large-scale plants. In particular, a cost-effective and reliable device, which should also be suitable for handling low and variable power, is fundamental.

Since some years, Pumps As Turbines (PATs), i.e., pumps employed in turbine mode, are considered a viable alternative to conventional hydraulic turbines, since their cost per nominal power can be one order of magnitude lower.

Several studies have evaluated the electrical energy production by means of PATs, by demonstrating that they are a reliable and versatile device that can be integrated within different environments, such as rural areas and Water Distribution Networks (WDNs).

For example, Chacón et al. [1] investigated the effectiveness of PATs within an irrigation network, by stating that the total energy recovery can be as high as $230 \mathrm{MWh}$. Rossi et al. [2] analysed the installation of a PAT in an aqueduct, by highlighting that the electrical energy production supplied by the selected turbomachine could be higher than $338 \mathrm{kWh}$ for each day of operation. In addition, the same authors discussed the installation of a PAT within a wastewater sewer of an oil refinery. In this case, the energy recovery throughout one year of operation was roughly equal to $19 \mathrm{MWh}$ [3].

However, PAT exploitation is generally discouraged by the fact that pump manufactures rarely provide performance curves of pumps running in reverse mode. Thus, several studies (e.g., [4-8]) have been addressed to tune proper models that predict both the Best Efficiency Point (BEP) and overall performance curves of PATs.

Another challenge related to PAT field exploitation is represented by the need of a procedure to select the best PAT to be installed in a given site in order to maximize electrical energy production. Several attempts have been documented in the literature in the last years and are reviewed in the following, to highlight the starting point of the study carried out in this paper.

Venturini et al. [9, 10] investigated several PAT-WDN combinations with the aim of selecting the best PAT on the basis of the pump BEP and the WDN features.

Several researchers identified the best pump running in reverse mode starting by the evaluation of the BEP of the PAT. For example, Stefanizzi et al. [11] and Barbarelli et al. [12] assumed that the flow rate and head at the BEP of the PAT are equal to the mean flow rate and head of the selected site, respectively. After that, the $\mathrm{BEP}$ of the pump running in direct mode was calculated by means of empirical equations that correlate pump and

\footnotetext{
* Corresponding author: lucrezia.manservigi@unife.it
} 
PAT operating modes. Finally, the BEP of the pump, in terms of head and flow rate, was used to select the optimal turbomachine. Similarly, Alberizzi et al. [13] set the BEP of the PAT equal to the design parameters of the WDN. Then, the pump running in direct mode was identified by means of empirical correlations that estimate both the specific speed and specific diameter of the considered turbomachine that, as a result, can be chosen from pump catalogues.

The model developed by Pugliese et al. [14] was aimed at estimating the BEP, the impeller diameter and the rotational speed of the PAT that maximizes the energy production. According to this study, the maximum energy production was achieved when the ratio between the maximum flow rate and the flow rate at the BEP of the PAT was equal to 0.951. Finally, the methodology proposed by Chacón et al. [15] selected the PAT that, among several alternatives, minimized the payback period.

In this framework, the current paper develops a novel comprehensive methodology that leads to the selection of the best PAT for a given site. The comprehensive methodology identifies the best turbomachine by means of only the BEP of the pump and the design parameters of the WDN. The novel methodology accounts for the physics behaviour of the PAT and differs from most of the previously mentioned approaches because the knowledge of the BEP and the performance curves of the PAT is not required.

\section{Methodology}

The methodology developed in this paper for selecting the best PAT, i.e., the one that maximizes energy production, for a given site comprises two steps. First, turbomachines whose energy production may be very low are preliminarily filtered out and thus a short list of PATs is defined among a pool of candidate PATs. Then, the most suitable machine is identified by means of a novel index that accounts for PAT-site matching.

\subsection{PAT short list}

In order to preliminarily filter out PATs that are not suitable to be installed in a given WDN, this paper develops two equations that correlate pump and PAT operating modes. The two equations estimate the minimum flow rate and head at which a given PAT can operate (namely runaway condition [16]) by means of the BEP of the pump.

The runaway condition is tuned in this paper by considering 21 centrifugal pumps, whose experimental data for both direct and reverse modes are available in the literature ([6], [7], [12], [17], [18]). The 21 turbomachines were selected so that the experimental data of both operating modes refer to the same rotational speed. For the considered turbomachines, Table 1 provides the number of the considered PATs, diameter $(D)$, pump and PAT rotational speed $(N)$, ranges of the pump BEPs (i.e., $Q_{\mathrm{BEP}}$ and $H_{\mathrm{BEP}}$ ) and operating ranges of the considered PATs.

For each turbomachine reported in Rossi et al. ([6]), Tan and Engeda ([7]) and Barbarelli et al. ([12]), the experimental data of each pump and PAT refer to only one rotational speed, i.e., $1050 \mathrm{rpm}$ or $1450 \mathrm{rpm}$. Instead, only one turbomachine is analysed by Stefanizzi et al. ([17]), but four different rotational speeds are considered (i.e., $800 \mathrm{rpm}, 1000 \mathrm{rpm}, 1200$ rpm and $1520 \mathrm{rpm})$. Similarly, Delgado et al. ([18]) provide the characteristics curves of three turbomachines for eight different rotational speeds, i.e., $600 \mathrm{rpm}, 900 \mathrm{rpm}, 1200 \mathrm{rpm}, 1500 \mathrm{rpm}, 1800 \mathrm{rpm}, 2100$ rpm, $2400 \mathrm{rpm}$ and $2700 \mathrm{rpm}$.

Thus, the runaway condition is identified by means of 45 experimental data sets in total, referring to 21 PATs and covering an extended range of head and flow rate.

The correlation between the flow rate at the experimental BEP of each pump (i.e., $Q_{\mathrm{BEP}, \mathrm{p}}$ ) and the experimental runaway flow rate of the PAT (i.e., $Q_{\mathrm{r}, \mathrm{t}}$ ) is shown in Fig. 1. As can be seen, experimental data exhibit an almost linear correlation (see Eq. (1)), whose coefficient of determination is equal to 0.98 .

$$
Q_{\mathrm{r}, \mathrm{t}}=0.5856 \cdot Q_{\mathrm{BEP}, \mathrm{p}}+2.0815
$$

Figure 2 sketches the correlation between head at the experimental BEP of each pump (i.e., $H_{\mathrm{BEP}, \mathrm{p}}$ ) and the experimental runaway head of the PAT running at the same rotational speed (i.e., $H_{\mathrm{r}, \mathrm{t}}$ ). As in Fig. 1, experimental data exhibit an approximately linear trend (see Eq. (2)), though the coefficient of determination (equal to 0.86) is lower than that of Eq. (1) mainly because of two PATs with $H_{\mathrm{BEP}, \mathrm{p}}$ equal to approximately $30 \mathrm{~m}$.

$$
H_{\mathrm{r}, \mathrm{t}}=0.9710 \cdot H_{\mathrm{BEP}, \mathrm{p}}-0.9877
$$

These results clearly demonstrate that pump and PAT operations are strictly correlated and the minimum operating condition of a PAT can be predicted only on the basis of the BEP of the pump, as discussed in [16].

The runaway condition can be exploited as follows, to define PAT short list. First, starting from the BEP of the pump, $Q_{\mathrm{r}, \mathrm{t}}$ and $H_{\mathrm{r}, \mathrm{t}}$ are obtained by means of Eqs. (1)

Table 1. Pump and PAT characteristics and operating ranges

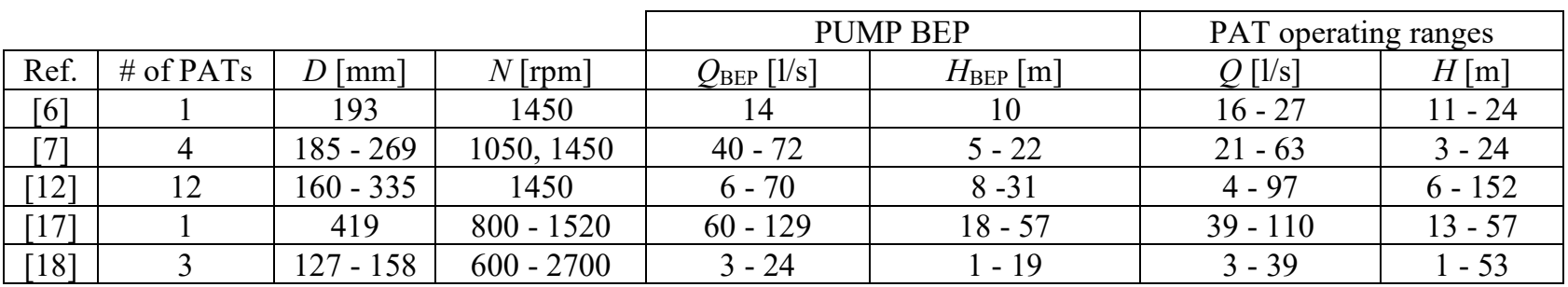




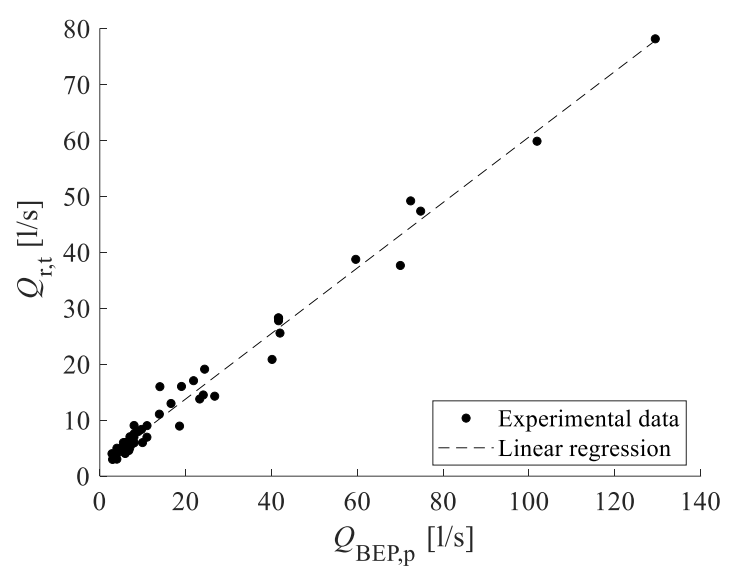

Fig. 1. Correlation between volume flow rate at pump BEP and runaway volume flow rate of the PAT

and (2) and are compared to the maximum volume flow rate and head of the considered WDN (i.e., $Q_{\max , W D N}$ and $\left.H_{\text {max,WDN }}\right)$. If the runaway condition exceeds at least one of the two parameters (Eqs. (3) or (4)), that PAT can be filtered out, since the electrical energy production will likely be negligible or even null.

$$
\begin{aligned}
& Q_{\mathrm{r}, \mathrm{t}}>Q_{\max , \mathrm{WDN}} \\
& H_{\mathrm{r}, \mathrm{t}}>H_{\text {max }, \mathrm{WDN}}
\end{aligned}
$$

Thus, the best PAT can be selected among the remaining turbomachines composing the short list.

\subsection{PAT selection}

The selection of the best PAT can be performed as follows. For each pump included in the short list, the

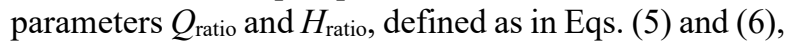
are calculated, in order to correlate the BEP of the pump and the design parameters of the WDN, which in this paper are set equal to the mean volume flow rate $\left(Q_{\text {mean,WDN }}\right)$ and head $\left(H_{\text {mean,WDN }}\right)$ of the site.

$$
\begin{aligned}
Q_{\text {ratio }} & =\frac{Q_{\mathrm{BEP}, \mathrm{p}}}{Q_{\text {mean, WDN }}} \\
H_{\text {ratio }} & =\frac{H_{\mathrm{BEP}, \mathrm{p}}}{H_{\text {mean, WDN }}}
\end{aligned}
$$

In a previous study, Venturini et al. [9] coupled four PATs to three WDNs and, for each case study, provided the $Q_{\text {ratio }}$ and $H_{\text {ratio }}$ that allowed to maximize the electrical energy production. The main outcome of the paper [9] was that the $Q_{\text {ratio }}$ should be roughly equal to 1, while the $H_{\text {ratio }}$ should range from 0.5 to 1.4 .

In the current paper, the best PAT is identified by means of a novel index, namely PAT-Site Index (PSI), defined in Eq. (7), which estimates the suitability of a given pump to run as a PAT in a given site.

$$
P S I=\sqrt{\left(Q_{\text {ratio }}-Q_{\text {ref }}\right)^{2}+\left(H_{\text {ratio }}-H_{\text {ref }}\right)^{2}}
$$

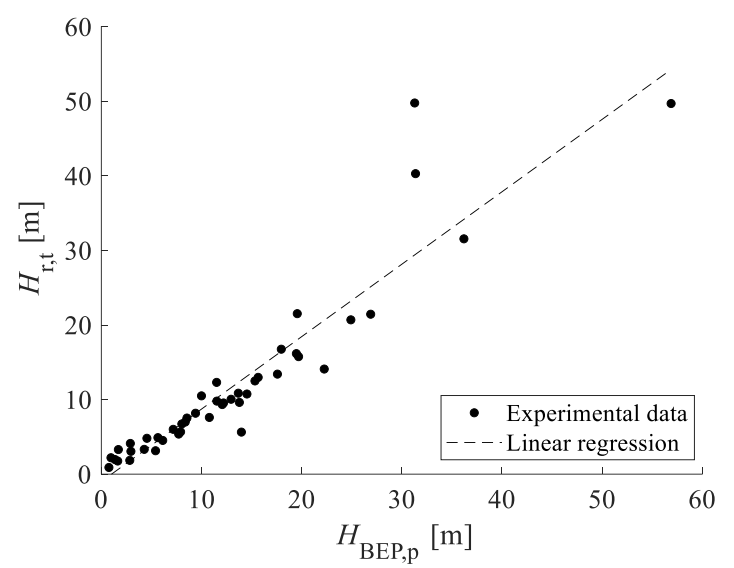

Fig. 2. Correlation between head at pump BEP and the runaway head of the PAT

Based on the guideline provided by Venturini et al. [9], $Q_{\text {ref }}$ is set equal to 1 , while $H_{\text {ref }}$ is equal to 0.95 , which is the mean value of the recommended range (i.e., from 0.5 to 1.4). As a consequence, the best PAT is the one that minimizes $P S I$, since it has to be as close as possible to the respective reference values.

\section{Case study}

In order to evaluate the effectiveness of the methodology described above, a simulated WDN is supposed to be coupled with the 21 PATs employed for tuning the runaway equations reported in Section 2.1.

All PAT performance curves, i.e., head, power and efficiency over the entire operating range, are modelled by interpolating the collected experimental data by means of a second-order polynomial function.

In addition, the producible electric power is calculated only within the experimental flow rate range in which each PAT was sampled. In fact, this assumption always guarantees PAT actual operation.

\subsection{Simulated site}

The site considered in this paper simulates a WDN in line with the experimental data reported in [10], in which four WDNs were considered.

In the current study, as in [10], data refer to one year of operation. Since the sampling frequency in [10] was equal to $15 \mathrm{~min}$, the simulated WDN accounts for 35,040 operating points.

The simulated WDN mimics one of the four WDNs documented in [10], in which field data were characterized by almost regular head drop measurements (average and standard deviation values are equal to $12 \mathrm{~m}$ and $2 \mathrm{~m}$, respectively), while the volume flow rate significantly varied over the year of operation, as shown in Fig. 3. In fact, the average volume flow rate and its standard deviation are equal to $116 \mathrm{l} / \mathrm{s}$ and $54 \mathrm{l} / \mathrm{s}$, respectively.

Data of the simulated WDN ( $Q_{\mathrm{WDN}}$ and $\left.H_{\mathrm{WDN}}\right)$ were obtained by considering the experimental volume flow 


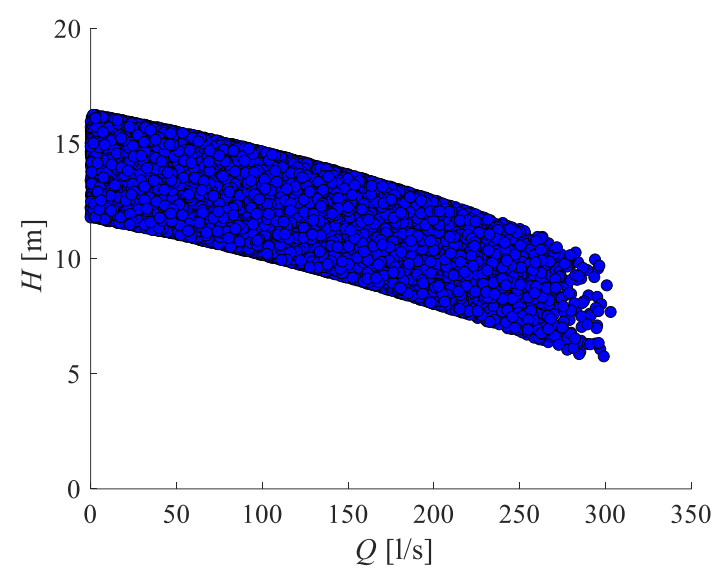

Fig. 3. Head drop $v s$. volume flow rate of the simulated WDN

rates $\left(Q_{\mathrm{e}}\right)$, head drop data $\left(H_{\mathrm{e}}\right)$ and the respective standard deviations (i.e., $\sigma_{\mathrm{Q}, \mathrm{e}}$ and $\sigma_{\mathrm{H}, \mathrm{e}}$ ), as follows:

- STEP 1: $H_{\mathrm{e}}$ data are interpolated by means of a second-order polynomial function, by achieving the interpolated function $H_{\text {int }}$ and the corresponding polynomial coefficients;

- STEP 2: for both head drop and volume flow rate, noise (i.e., noise $_{\mathrm{Q}, \mathrm{i}}$ and noise $_{\mathrm{H}, \mathrm{i}}$ ) is randomly generated in the range $-\sigma$ and $+\sigma$ for both experimental flow rate and head;

- STEP 3: each simulated volume flow rate (i.e., $\left.Q_{\mathrm{WDN}, \mathrm{i}}\right)$ is calculated by adding each experimental volume flow rate (i.e., $Q_{\mathrm{e}, \mathrm{i}}$ ) and the volume flow rate noise (i.e., noise $_{\mathrm{Q}, \mathrm{i}}$ );

- STEP 4: $Q_{\mathrm{WDN}, \mathrm{i}}$ feeds the second-order polynomial function obtained in STEP 1. The output of this step is the corresponding interpolated head drop value (i.e., $H_{\text {int,i }}$;

- STEP 5: finally, the simulated head drop (i.e., $\left.H_{\mathrm{WDN}, \mathrm{i}}\right)$ is calculated by adding $H_{\mathrm{int,i}}$ and the randomly generated head drop noise (i.e., noise $_{\mathrm{H}, \mathrm{i}}$ ).

STEP 2 through STEP 5 are repeated for all the 35,040 data, to obtain the simulated WDN.

Figure 3 shows the simulated WDN, in which the volume flow rate ranges from $0 \mathrm{l} / \mathrm{s}$ to $303 \mathrm{l} / \mathrm{s}$ and the mean volume flow rate and standard deviation are equal to $117 \mathrm{l} / \mathrm{s}$ and $60 \mathrm{l} / \mathrm{s}$, respectively. As for the experimental WDN reported in [10], the head drop exhibits an almost constant trend, ranging from $6 \mathrm{~m}$ to $16 \mathrm{~m}$, while the average head is equal to $12 \mathrm{~m}$ and the standard deviation is slightly lower than $2 \mathrm{~m}$.

\subsection{Electrical energy production}

In this paper, the electrical energy production by means of a PAT is calculated by assuming a hydraulic regulation that comprises (i) the dissipation of the exceeding head and (ii) the reduction of volume flow rate [10]. This assumption relies on the fact that the hydraulic regulation allows the minimization of both installation and regulation costs, since it only requires two valves, so that the PAT is in series with one valve, while it is in parallel with the other valve. The valve-PAT in series layout has the aim of dissipating the exceeding pressure of the WDN, according to Eq. (8).

$$
\text { if } H_{\mathrm{PAT}} \leq H_{\mathrm{WDN}} \text { then } \Delta H_{\mathrm{un}}=H_{\mathrm{WDN}}-H_{\mathrm{PAT}}
$$

For a given flow rate $\left(Q_{\mathrm{WDN}}\right)$, if the head drop of the WDN $\left(H_{\mathrm{WDN}}\right)$ exceeds the PAT corresponding value $\left(H_{\mathrm{PAT}}\right)$, the difference between the head drop values will be dissipated and thus unexploited $\left(\Delta H_{\mathrm{un}}\right)$. Then, PAT producible power is calculated at $Q_{\mathrm{WDN}}$.

Instead, the valve-PAT in parallel layout allows the reduction of the volume flow rate feeding the PAT, in the case that PAT head drop exceeds the corresponding WDN value (Eq. (9)). The flow rate of the WDN can be reduced up to $Q_{\mathrm{PAT}}$, depending on the head curve of the PAT. The unexploited volume flow rate $\left(\Delta Q_{\text {un }}\right)$ can be calculated as in Eq. (9).

$$
\text { if } H_{\mathrm{PAT}}>H_{\mathrm{WDN}} \text { then } \Delta Q_{\mathrm{un}}=Q_{\mathrm{WDN}}-Q_{\mathrm{PAT}}
$$

Then, PAT producible power is calculated at $Q_{\text {PAT }}$.

Finally, the producible electrical energy is calculated by multiplying the producible electric power by the sampling time of WDN data. It is worth highlighting that this paper provides estimates of the maximum electrical energy production, since the mechanical-to-electric energy conversion efficiency is set equal to 1 .

\section{Results}

This Section discusses the effectiveness of the methodology to identify the best PAT to install in the considered WDN.

The first step of the methodology consists of the application of Eq. (1) through (4), i.e., the runaway condition. This check correctly allows to discard 11 out of the 45 data sets. In fact, by further investigating these 11 data sets, it was found out that energy production ranges from 0 to approximately $800 \mathrm{kWh} /$ year. Moreover, these 11 data sets were also double-checked by applying the guidelines provided by Venturini et al. in [9]. In fact, the $H_{\text {ratio }}$ of these 11 data sets is higher than 1.5 , while it should be lower than 1.4 according to [9]. Such agreement is a relevant outcome, since the current paper and [9] consider different PATs.

The second step of the methodology requires the evaluation of the PSI to further discriminate between the remaining 34 data sets. As shown in Fig. 4, which reports the electrical energy production vs. the PSI of the 34 data sets, electrical energy production exhibits a decreasing trend by increasing PSI. In particular, both the best PAT (green symbol) and second-best PAT (yellow symbol) are highlighted. It is worth noting that the PSI unequivocally and successfully identifies the best PAT, since the maximum electrical energy production is achieved by considering the lowest PSI, i.e., 0.45 .

\subsection{Best PAT}

The BEP of the best pump is reported below:

$$
\text { - } Q_{\mathrm{BEP}, \mathrm{p}}=70 \mathrm{l} / \mathrm{s} \text {; }
$$




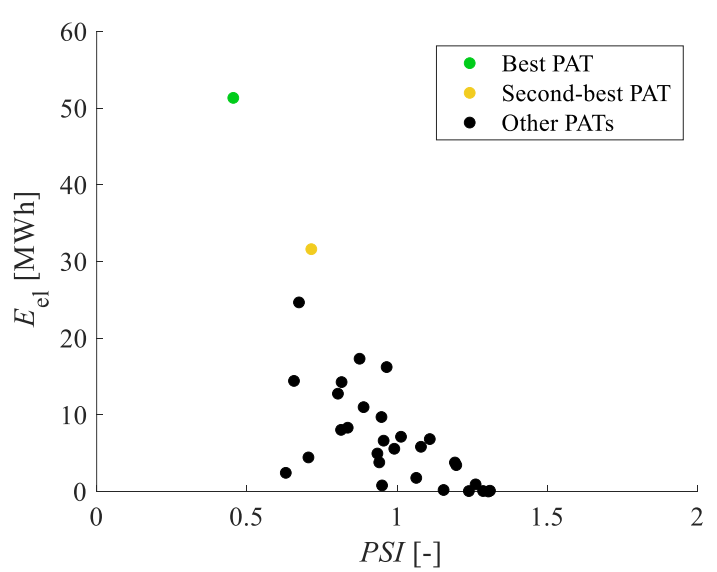

Fig. 4. Electrical energy production vs. PSI

- $H_{\mathrm{BEP}, \mathrm{p}}=14 \mathrm{~m}$.

The best PAT operates with an average efficiency of $81 \%$ and allows the production of $51 \mathrm{MWh} /$ year. Such energy production represents a promising result, since it may be even further increased by considering a larger number of PATs and/or investigating different regulation strategies, as demonstrated in [13].

The successful PAT selection is also conveyed by means of Fig. 5, in which dark/light-blue and grey symbols represent the WDN data that can and cannot be exploited, respectively, by means of the best PAT, while red crosses represent PAT operating points.

As can be seen, the large majority of WDN data (approximately $88 \%$ ) can be exploited for electrical energy production, of which $20 \%$ undergo a head drop dissipation (light-blue symbols in Fig. 5). As a result, energy loss because of head dissipation is approximately equal to $5.6 \mathrm{MWh} /$ year. Instead, $80 \%$ of the exploitable flow rate is throttled (dark-blue symbols in Fig. 5), by causing an energy loss of approximately $55 \mathrm{MWh} /$ year.

Thus, the installation of an additional PAT, in parallel with the best turbomachine, could be a viable solution for further enhancing electrical energy production. In such a manner, the throttled flow rate could be also exploited.

Finally, it is worth highlighting that some PAT-site combinations allowed the exploitation of more than $88 \%$ of the available WDN data. However, in spite of this, electrical energy production was not maximized; in

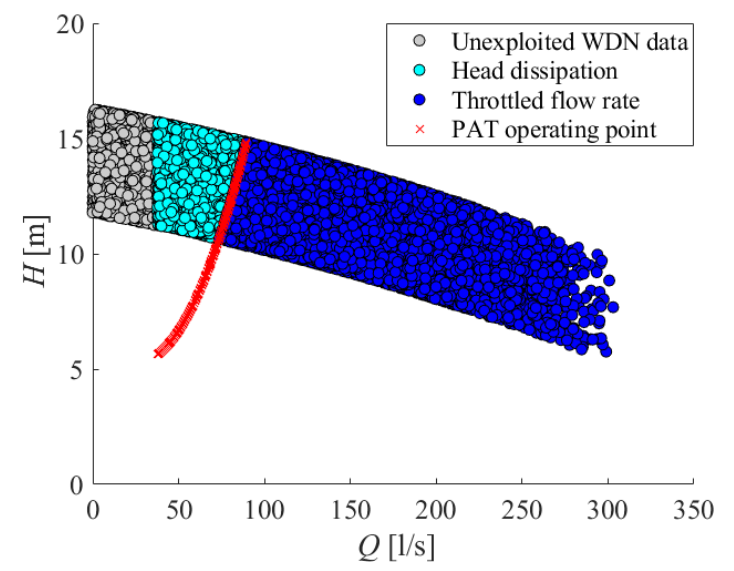

Fig. 5. WDN data compared to the performance curve of the best PAT fact, it was at least $66 \%$ lower than the electrical energy which can be produced by using the best PAT.

\subsection{Second-best PAT}

Figure 6 depicts the exploitation of WDN data by means of the second-best PAT, which produces $32 \mathrm{MWh} /$ year. In such a case, $87 \%$ of WDN data can be exploited for energy production. As for the best PAT, throttled flow rate leads to higher energy losses, which are equal to $73 \mathrm{MWh} /$ year, while approximately $3 \mathrm{MWh} /$ year are lost because of head dissipation.

As can be seen, second-best PAT operating points (i.e., red crosses) reported in Fig. 6 do not exploit the WDN data whose flow rates are higher than $63.0 \mathrm{l} / \mathrm{s}$ and head higher than $14.1 \mathrm{~m}$. Such limitation relies on the operating range on which the second-best PAT was sampled. Conversely, best PAT operating points (see Fig. 5) allow to exploit all WDN data whose flow rate and head are higher than $37.7 \mathrm{l} / \mathrm{s}$ and $5.6 \mathrm{~m}$, respectively. In fact, such PAT was sampled up to $97.0 \mathrm{l} / \mathrm{s}$ and $17.2 \mathrm{~m}$.

\subsection{Hydraulic energy exploitation}

Energy production over one year by means of the best and second-best PAT is reported in Fig. 7, by highlighting that the best PAT and second-best PAT allow the exploitation of $45 \%$ and $28 \%$, respectively, of the available WDN hydraulic energy.

\section{Conclusions}

This paper dealt with the development of a novel comprehensive methodology aimed at selecting the best Pump As Turbine (PAT) that, among several alternatives, allows the maximization of electrical energy production.

The methodology comprises two steps and only requires the knowledge of the best efficiency point of the considered pumps and the hydraulic parameters of the considered site.

First, for each pump, the runaway condition is evaluated by means of two equations developed by the authors based on the experimental data of PATs

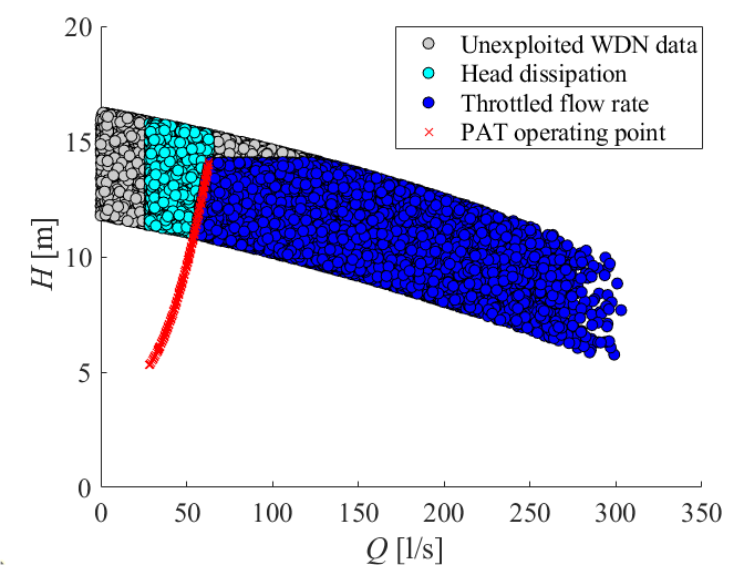

Fig. 6. WDN data compared to the performance curve of the second-best PAT 


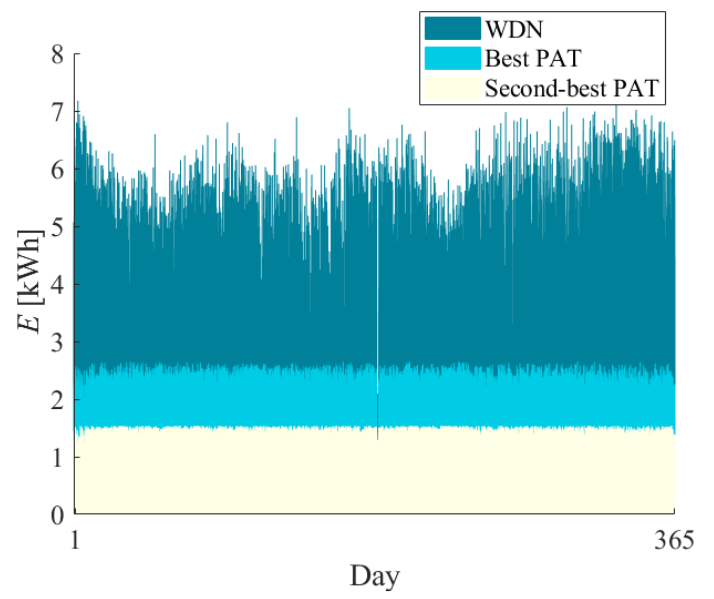

Fig. 7. Hydraulic energy of the WDN compared to electrical energy production by means of the best and second-best PAT

available in the literature. For all pumps whose runaway condition fulfils the maximum hydraulic parameters of the site, a novel index, namely PAT-Site Index, is calculated. According to the index definition, the best PAT is the one that allows the minimum PAT-Site Index.

The novel methodology was tested by calculating the electrical energy production of 21 centrifugal PATs, of which the experimental performance curves were derived from the literature, also available at different rotational speeds. The PATs were coupled with a simulated site, representative of a water distribution network. The runaway condition allowed to filter out most of PATs whose energy production was negligible or equal to zero. In addition, the minimum PAT-Site Index successfully identified the best PAT.

Future analyses are planned to further test the novel methodology, by investigating additional combinations of both PATs and sites.

\section{References}

1. M.C. Chacón, J.A.R. Díaz, J.G. Morillo, A. McNabola, Renew. Energy 147, 1728 (2020)

2. M. Rossi, M. Righetti, M. Renzi, Energy Procedia 101, 1207 (2016)

3. M. Renzi, P. Rudolf, D. Štefan, A. Nigro, M. Rossi, Energy recovery in oil refineries through the installation of axial Pumps-as-Turbines (PaTs) in a wastewater sewer: a case study, 10th International Conference on Applied Energy, ICAE 2018, 22-25 August 2018, Hong Kong, China (2018)

4. M. Venturini, L. Manservigi, S. Alvisi, S. Simani, Appl. Energy 231, 343 (2018)

5. M. Stefanizzi, M. Torresi, B. Fortunato, S. Camporeale, Energy Procedia 126, 589 (2017)

6. M. Rossi, A. Nigro, M. Renzi, Appl. Energy 248, 555 (2019)

7. X. Tan, A. Engeda, Renew. Energy 99, 188 (2016)

8. L. Manservigi, M. Venturini, E. Losi, Application of a physics-based model to predict the performance curves of pumps as turbines, AIP Conference Proceedings 2191, 020106 (2019)

9. M. Venturini, S. Alvisi, S. Simani, Energy potential of pumps as turbines (PATs) in water distribution networks, in Proceedings of the International Conference on Efficiency, Cost, Optimization, Simulation and Environmental Impact of Energy Systems, ECOS, 30 June - 3 July 2015, Pau, France (2015)

10. M. Venturini, S. Alvisi, S. Simani, L. Manservigi, Energies 11, 1016 (2017)

11. M. Stefanizzi, T. Capurso, G. Balacco, M. Binetti, M. Torresi, S.M. Camporeale, Pump as turbine for throttling energy recovery in water distribution networks, AIP Conference Proceedings 2191, 020142 (2019)

12. S. Barbarelli, M. Amelio, G. Florio, Energy Convers. Manag. 149, 781 (2017)

13. J.C. Alberizzi, M. Renzi, M. Righetti, G.R. Pisaturo, M. Rossi, Energies 12, 4738 (2019)

14. F. Pugliese, F. De Paola, N. Fontana, G. Marini, M. Giugni, Optimal Selection of Pumps As Turbines in Water Distribution Networks, Proceedings 2, 685 (2018)

15. M.C. Chacón, J.A.R. Díaz, J.G. Morillo, A. McNabola, Water 11, 149 (2019)

16. J.F. Gulich, Centrifugal pumps. Springer $3^{\text {rd }}$ Edition 2010. Berlin Heidelberg

17. M. Stefanizzi, T. Capurso, G. Balacco, M. Torresi, M. Binetti, A.F. Piccinni, B. Fortunato, S.M. Camporeale, Preliminary assessment of a pump used as turbine in a water distribution network for the recovery of throttling energy, Proceedings of 12th European Conference on Turbomachinery Fluid dynamics \& Thermodynamics ETC13, April 8-12 2018, Lausanne, Switzerland (2018)

18. J. Delgado, J.P. Ferreira, D.I.C. Covas, F. Avellan, Renew. Energy 142, 437 (2019) 